\title{
Palmoplantar Pustulosis-like Eruption Induced by Baricitinib for Treatment of Rheumatoid Arthritis
}

\author{
Dimitra Koumaki ${ }^{1}$, Vasiliki Koumaki ${ }^{2}$, Eleni Lagoudaki ${ }^{3}$, George Bertsias ${ }^{4}$ \\ ${ }^{1}$ Dermatology Department, University Hospital of Heraklion, Crete, Greece \\ ${ }^{2}$ Microbiology Department, Medical School of Athens, Athens, Greece \\ ${ }^{3}$ Pathology Department, University Hospital of Heraklion, Crete, Greece \\ ${ }^{4}$ Rheumatology Department, University Hospital of Heraklion, Crete, Greece
}

Received: 11/11/2019

Accepted: 23/11/2019

Published: $19 / 12 / 2019$

How to cite this article: Koumaki D, Koumaki V, Lagoudaki E, Bertsias G. Palmoplantar pustolosis-like eruption by baricitinib for treatment of rheumatoid arthritis. EJCRIM 2019;7: doi:10.12890/2019_001383.

Conflicts of Interests: The Authors declare that there are no competing interest

This article is licensed under a Commons Attribution Non-Commercial 4.0 License

\section{ABSTRACT}

Objectives: Baricitinib is an orally active Janus kinase (JAK) inhibitor used in the treatment of moderate to severe rheumatoid arthritis (RA). Materials and methods: Here, we report the case of a 56-year-old Caucasian male diagnosed with RA who developed palmoplantar pustulosis (PPP) while being treated with baricitinib.

Results: The patient's PPP resolved after discontinuation of baricitinib and recurred when this was restarted. Based on causality assessment, it was considered a drug-induced PPP.

Conclusion: To the authors' knowledge, this is the first case of baricitinib-induced PPP.

\section{LEARNING POINTS}

- Baricitinib is a small, orally active molecule that inhibits JAK-1 and JAK-2, which is used in the treatment of rheumatoid arthritis.

- Baricitinib has been also used in the treatment of psoriasis, alopecia areata and atopic dermatitis.

- Palmoplantar pustulosis is a rare cutaneous side effect of baricitinib

\section{KEYWORDS}

Palmoplantar pustulosis, baricitinib, rheumatoid arthritis, cutaneous side effects

\section{INTRODUCTION}

Baricitinib is an orally administered, small-molecule JAK-1 and -2 inhibitor that is used in the treatment of rheumatoid arthritis (RA) $)^{[1]}$. Here, we report the first known case of palmoplantar pustulosis (PPP)-like eruption following baricitinib treatment for RA.

\section{CASE DESCRIPTION}

A 56-year-old Caucasian male was diagnosed with seropositive erosive RA 7 years previously. His past medical history was otherwise unremarkable. He had been treated with multiple disease-modifying antirheumatic drugs (DMARDs), including methotrexate and leflunomide. In 2014, after failure of the aforementioned DMARDs, he was started on oral baricitinib 4 mg once daily, which he was stable on for 5 years. Five years after having started baricitinib, he developed hyperkeratosis, fissuring and pustules on both palms and soles. $A$ dermatology referral was made at that point. Clinical examination revealed thickened scaly skin with pustules on both the palms and soles (Fig. 1). The histology of a skin biopsy from the affected areas was in keeping with palmoplantar pustular psoriasis (Fig. 2). 


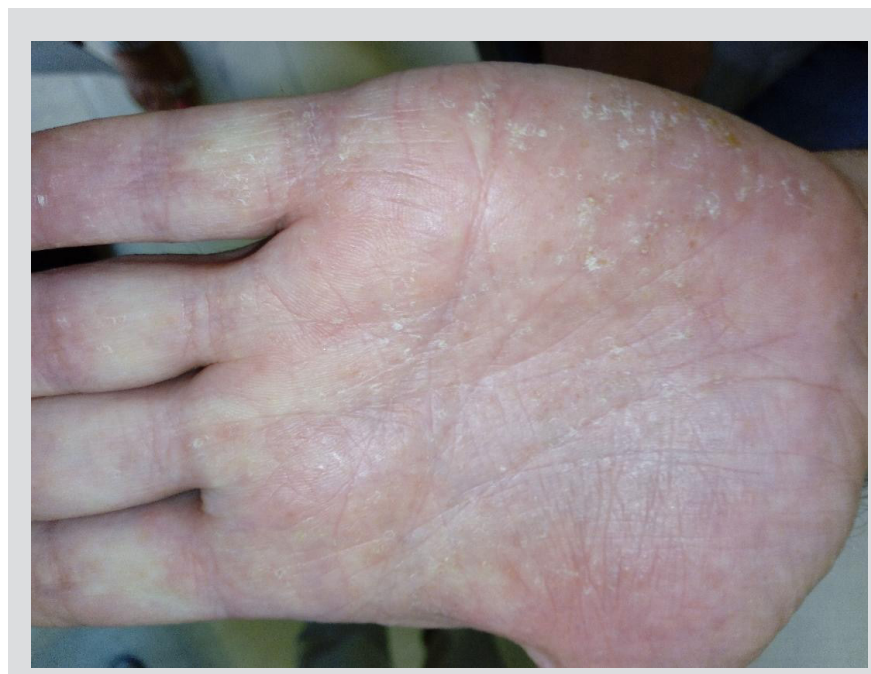

Figure 1. Pustules on the affected palms

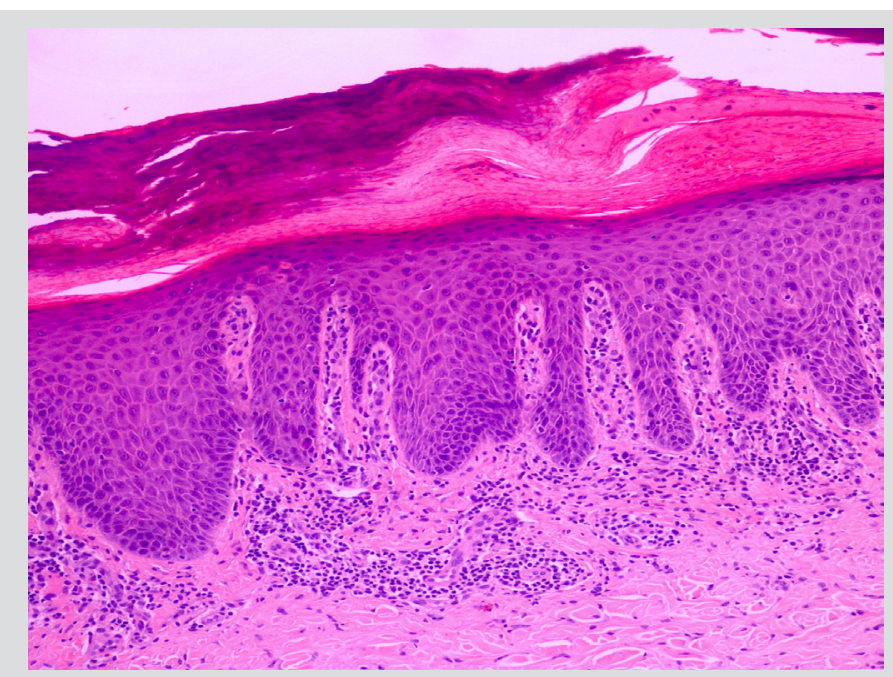

Figure 2. Histology of the skin biopsy revealed confluent parakeratosis, acanthosis, neutrophils in stratum corneum and a dermal infiltrate of lymphocytes (haematoxylin and eosin stain, x20 magnification).

The patient was seen by a dermatologist who suspected palmoplantar pustular psoriasis based on clinical and histologic findings. There was no past medical history nor family history of psoriasis or atopic dermatitis. Baricitinib was discontinued for 2 months to rule out its aetiological involvement in the palmoplantar pustular psoriasis. The patient was also prescribed topical treatment with emollients to apply twice daily and topical clobetasol propionate $0.05 \%$ ointment to use twice daily for 14 days and then every other day for 2 more weeks. After 2 months of follow-up, there was an improvement of the patient's skin condition with near complete clearance. However, because of deterioration of his RA, baricitinib $4 \mathrm{mg}$ once daily was restarted. Subsequently, the PPP reappeared on both his palms and soles after 14 days. Based on the clinical course of events, we diagnosed PPP-like eruption due to baricitinib treatment. Baricitinib dosage was lowered to $2 \mathrm{mg}$ once daily, which was more tolerable for his skin condition. The patient remains under dermatology and rheumatology long-term follow-up.

\section{DISCUSSION}

Baricitinib is a small, orally active drug available in the United States ( $2 \mathrm{mg} / \mathrm{day}$ ), the European Union ( 2 and $4 \mathrm{mg} / \mathrm{day}$ ) and other countries, that preferentially inhibits JAK-1 and JAK- $2^{[1]}$. It is indicated for the treatment of moderate to severe active RA in adult patients who have responded inadequately to, or who are intolerant to, 1 or more DMARDs ${ }^{[1]}$. It has also been used in clinical trials for the treatment of atopic dermatitis and psoriasis ${ }^{[1]}$. Other JAK inhibitors such as tofacitinib, a JAK-1/3 inhibitor, are actually used in the treatment of PPP rather than causing disease ${ }^{[2,3]}$. Recently, a case was published regarding tofacitinib causing PPP ${ }^{[4]}$. It is well established that TNF- $\alpha$ inhibitors can cause PPP as a paradoxical effect. In our case, based on the clinical course of events, baricitinib was the cause of PPP. The underlying pathophysiology of this reaction is uncertain; psoriasis is thought to develop secondary to an abnormal T cell response where several cytokines are implemented such as IL-12, IL-17 and IL-23 ${ }^{[5]}$. These have been shown to activate the JAK/STAT pathway ${ }^{[5]}$. IL-23 is closely associated with JAK-2 ${ }^{[5]}$. JAK-1/3 inhibition may downregulate IL-1 and IL-8, which are potential mediators of PPP ${ }^{[3]}$. In conclusion, more studies investigating the pathways involved in PPP need to be carried out, including baricitinib-induced PPP and the effects of the drug on the $T$ cell-mediated immune response.

This case highlights a new side effect of baricitinib, a JAK inhibitor, namely PPP-like eruption.

\section{REFERENCES}

1. Damsky W, King BA. JAK inhibitors in dermatology: the promise of a new drug class. J Am Acad Dermatol 2017;76(4):736-744.

2. Koga T, Sato T, Umeda M, Fukui S, Horai Y, Kawashiri SY, et al. Successful treatment of palmoplantar pustulosis with rheumatoid arthritis, with tofacitinib: impact of this JAK inhibitor on T-cell differentiation. Clin Immunol 2016;173:147-148.

3. Haynes D, Topham C, Hagstrom E, Greiling T. Tofacitinib for the treatment of recalcitrant palmoplantar pustulosis: a case report. Australas J Dermatol. Epub 2019 Jul 18. doi: 10.1111/ajd.13117.

4. Shibata T, Muto J, Hirano Y, Takama H, Yanagishita T, Ohshima Y, et al. Palmoplantar pustulosis-like eruption following tofacitinib therapy for juvenile idiopathic arthritis. JAAD Case Rep 2019;5:518-521.

5. Gündüz Ö. JAK/STAT pathway modulation: does it work in dermatology? Dermatol Ther 2019;32:e12903. 